\title{
Rough 集上不易辨识关系的特征性质
}

\author{
施 恩 伟 \\ （云南师范大学, 昆明)
}

\section{关铮词 Rough 集、信息系统、不易辨识关系}

Rough 集是波兰学者 Pawlak 1981 年提出的一个新概念. 由于该理论在信息系统及人 机系统等许多领域中有着广泛的应用, 因而引起了国际上很多学者的关注 ${ }^{[1-3]}$, 经过短短几年 时间的研究,就已形成了一个应用广泛的新兴学科. 但是,该理论在作为刻划信息系统的理论 工具时,其主要理论基础即元素间的不易辨识关系是建立在信息函数值相等的等量关系上的. 然而,在实际问题中,信息量往往是不容易确定的. 为此, 本文以模糊等价关系作为建立不易 就识关系的理论基础,获得了许多重要的结果. 从而推广了 Pawlak 等人所得到的结果, 并且 扩大了该理论的应用范围.

设 $U$ 为非空集, $R$ 是 $U$ 上的等价关系. $A=(U, R)$ 称为近似空间. 令 $X \subset U$, 则

$$
\begin{aligned}
& \underline{R} X=\left\{x \mid x \in U,[x]_{R} \subset X\right\}, \\
& \bar{R} X=\left\{x \mid x \in U,[x]_{R} \cap X \neq \phi\right\} .
\end{aligned}
$$

分别称为 $X$ 关于 $A$ 的 $R$ 下界集及 $R$ 上界集, $[x]_{R}$ 是 $x$ 所在的等价类. 若 $\tilde{R} X-R X \neq \phi$, 则 称 $X$ 是关于 $R$ 的 Rough 集.

定义 1 一个信息系统是一个有序四重组

$$
F=(U, Q, V, f),
$$

夰中 $U$ 是论域, $Q$ 是特征集, $V-\bigcup_{a \in U} V_{q}$ 是映射 $f$ 的值域, $f: U \times Q \rightarrow V$ 叫做信息函数, 而 且对 $\forall x \in U, \forall q \in Q$, 有 $f(x, q) \in V_{q}$.

定义 2 设 $G$ 是 $V_{q}(q \in Q)$ 上的模糊等价关系, 对 $x, y \in U$, 若 $G(f(x, q), f(y, q)) \geqslant \alpha$, $\alpha \in(0,1]$, 则称 $x$ 与 $y$ 是 $\alpha$ 级不易辩识的, 记为 $x q_{a}^{*} y$.

定理 1 设 $G$ 是 $V$, 上的模糊等价关系, 则 $q_{a}^{*}$ 是 $U$ 上的普通的等价关系, $a \in(0,1]$.

证 因为 $G$ 是模糊等价的, 由 $G(f(x, q), f(x, q))-1$, 知 $x q_{a}^{*} x$ : 由 $G(f(x, q), f(y$, $q))-G(f(y, q), f(x, q))$, 知 $x q^{*}$ : 当且仅当 $y q_{a}^{*} x$, 而传递性也是易证的.

显然, 当 $\alpha 、 \beta \in(0,1], \alpha \leqslant \beta$ 时, $q_{3}^{*} \subset q_{a}^{*}$. 在本文只对两个确定的 $\alpha$ 进行讨论, 故简记 $x q_{a}^{*} y$ 为 $x q^{*} y$.

定义 3 设 $P \subset Q, x, y \in U$, 若对 $\forall q \in P$ 都有 $x q^{*} y$, 则称 $x$ 与 $y$ 关于 $P$ 是不易辩识 的, 记为 $x P^{*} y$. 约定 $\phi^{*}-U \times U$.

定义 4 我们称子集 $P \subset Q$ 关于系统 $F$ 是无关的, 如果对每个 $P_{0} \subset P, P_{0} \neq P$ 都有 $P^{*} \neq$ $P_{0}^{*}$ ，记为 $P \# F$. 如果存在某个 $P_{0} \subset P, P_{0} \neq P$ 使得 $P_{0}^{*}=P^{*}$, 则称 $P$ 关于 $F$ 是相关的, 记为:

本文 1989 年 2 月 3 日收到. 
$P \infty F$.

定理 2 设 $P \subset Q$, 若 $P \# F$, 则对每个 $P_{0} \subset P$ 都有 $P_{0} \# F$ : 若 $P \infty F$, 则对每个 $P_{0} \supset F$ 都 有 $P \infty F$.

证 若存在 $P_{0}$ 使得 $P_{0} \infty F$, 则存在 $P^{\prime} \subset P_{0}, P^{\prime} \neq P_{0}$ 使得 $P^{\prime *}-P_{0}^{*}$. 但 $P^{\prime} \subset P_{0} \subset P$, 所以 $P \infty F$, 这与 $P \# F$ 矛盾. 后一结论是明显的.

记 $E^{\prime}=\left\{P^{*} \mid P \subset Q\right\}, E-E^{\prime} /=$, 并仍用 $E^{\prime}$ 的元素的记号表示 $E$ 的元素. 令 $P \subset Q$ 且 $P=\bigcup_{q \in P}\{q\}$,因为 $\{q\}^{*} \subset U \times U$, 由 $P^{*}$ 的定义知 $P^{*}-\bigcap_{q \in P}\{q\}^{*}$. 因此, 对任意的 $P^{*} 、 K^{*} \epsilon$ $E$, 可知 $(P \cup K)^{*}-P^{*} \cap K^{*}$. 因而, 若 $P \subset K \subset Q$, 则 $Q^{*} \subset K^{*} \subset P^{*}$ 、为加以区别, 我们把 $P^{*} \subset$ $K^{*}$ 记为 $P^{*} \leqslant K^{*}$, 显然, $\leqslant$ 是 $E$ 上的偏序关系， $\phi^{*}$ 和 $Q^{*}$ 分别是最大和最小元.

定理 3 设 $P, K \subset S \subset Q$. 若 $S \# F$ 且 $P^{*} \subset K^{*}$, 则 $K \subset P$.

证 由 $P^{*} \subset K^{*}$ 知, $P^{*}-P^{*} \cap K^{*}-(P \cup K)^{*}$, 若 $P \neq P \cup K$, 因 $P \subset P \cup K$, 从而 $(P \cup$ $K) \infty F$, 这与定理 2 矛盾. 以上的结果是建立在定理 1 的基础之上的.

显然,我们可以在 $E$ 上定义二元运算 $\vee$ 及 $\Lambda$.

定义 5 对 $\forall P^{*}, K^{*} \in E$ 规定 $P^{*} \wedge K^{*}-(P \cup K)^{*}, P^{*} \vee K^{*}-(P \cap K)^{*}$.

上述定义中, $\vee$ 运算已不是通常意义下的集合取并运算. 但易证运算 $\vee$ 及 $\wedge$ 仍满足幂等 律、交换律、结合律及吸收律, 故 $(E, V, \Lambda)$ 是格. 记 $A_{*}^{*}-\bigvee_{i=1}^{*} P_{i}^{*}$, 易证 $\lim _{n \rightarrow \infty} A_{*}^{*}$ 存在. 定义 $\lim _{n \rightarrow \infty} A_{n}^{*}-\bigvee_{i=1}^{\infty} P_{i}^{*}$, 则易把 $\vee$ 和 $\wedge$ 推广到无限的情形. 由 $Q$ 的䓪集格是完全分配格这一事实, 我们可以得到定理 4 .

定现 4 代数系 $(E, V, \Lambda)$ 是完全分配格.

证 以 $I$ 表指标集. 对

$$
\begin{aligned}
& A_{i j}^{*} \in E, \bigwedge_{i \in i}\left(\bigvee_{i \in J_{i}} A_{i}^{*}\right)=\bigwedge_{i \in I}\left(\bigcap_{i \in J_{i}} A_{i j}\right)^{*}=\left[\bigcup_{i \in I}\left(\bigcap_{i \in J_{i}} A_{i j}\right)\right]^{*}=\left[\bigcap_{i \in \prod_{i \in l}}\left(\bigcup_{i \in I} A_{i f(i)}\right)\right]^{*}
\end{aligned}
$$

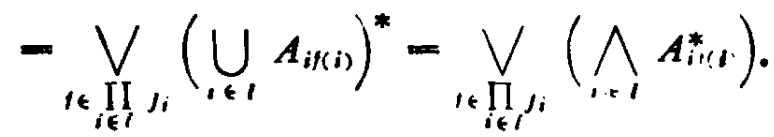

同理,可证完全分配格定义中的另一等式成立.

我们用 $L-(P(Q), U, \cap)$ 表示 $Q$ 的幕集格, 定义映射 $f: L \rightarrow E$ 为 $\forall P \in L, f(P)-P^{*}$, 则 $f$ 是对偶格同态,进而有

定理 $5 Q \# F$ 当且仅当 $L$ 与 $E$ 是对偶格同构。

证显然 $f$ 是满射, 由定义 4 及定理 3 知, $Q$ \# $F$ 当且仅当 $f$ 是单射, 因而是对偶格同 构.

如果对 $\forall P^{*} 、 B^{*} \in E$ 定义 $P^{*} \triangle B^{*}-\sup \left\{A^{*} \mid A^{*} \in E, P^{*} \wedge A^{*} \leqslant B^{*}\right\}$, 则有

性瓜 1 设 $F^{*} \in E$, 若 $B \subset Q$ 且 $B \# F$, 则 $P^{*} \triangle B^{*}-(B-P)^{*}$; 若 $Q \# F$, 则 $P^{*} \triangle$ $Q *$ 是 $P^{*}$ 的补元.

证 只证后一结论. 显然, $P^{*} \wedge\left(P^{*} \triangle Q^{*}\right)=P^{*} \wedge(Q-P)^{*}-(P \cup(Q-P))^{*}-$ $Q^{*} . P^{*} \vee\left(P^{*} \triangle Q^{*}\right)-P^{*} \vee(Q-P)^{*}-\phi^{*}$.

根据文献 [4]的定义 3 及命题 1 与命题 2 , 记 $B^{*} \circ P^{*}-P^{*} \triangle B^{*}$, 则有 
定理 6 代数系 $\left(E, 0, \phi^{*}\right)$ 是 $B C K$ 代数.

性质 2 对 $\forall A^{*}, B^{*}, C^{*} \in E$ 有 (1) $A^{*} \Delta\left(B^{*} \triangle C^{*}\right)=B^{*} \triangle\left(A^{*} \triangle C^{*}\right):(2) A^{*} \leqslant$ $B^{*} \triangle C^{*}$ 些且仅当 $B^{*} \leqslant A^{*} \triangle C^{*}$ ：（3） $T \phi^{*}=Q^{*}, T Q^{*}=\phi^{*}$ ：（4） $T\left(T A^{*}\right) \geqslant A^{*}$ ， $T\left(T\left(T A^{*}\right)\right)-T A^{*}$; (5) $T A^{*} \triangle T B^{*} \geqslant B^{*} \triangle A^{*}$; (6) $A^{*} \triangle T B^{*}=B^{*} \triangle T A^{*}$. 这里 $T A^{*}=A^{*} \triangle Q^{*}$, 对 $\forall A^{*} \in E$.

该性质由文献 [4]的结果立即可证.

例. 考虑信息系统 $F=(U, Q, V, f)$, 这里 $U=\left\{x_{1}, x_{2}, x_{3}, x_{1}, x_{5}\right\} ; Q=\{q, r\}$ 且 $f\left(x_{1}\right.$, $q)-0, f\left(x_{2}, q\right)-4, f\left(x_{3}, q\right)-1, f\left(x_{4}, q\right)=2.5, f\left(x_{j}, q\right)-2.8$. 记 $f_{i}-f\left(x_{i}, q\right)$, 我们假 定 $f_{i}(i-1,2,3,4,5)$ 之间的模糊关系矩阵如下:

\begin{tabular}{c|lllll} 
& $f_{1}$ & $f_{2}$ & $f_{3}$ & $f_{4}$ & $f_{5}$ \\
\hline$f_{1}$ & 1 & 0.1 & 0.8 & 0.5 & 0.3 \\
$f_{2}$ & 0.1 & 1 & 0.1 & 0.2 & 0.4 \\
$f_{3}$ & 0.8 & 0.1 & 1 & 0.3 & 0.1 \\
$f_{3}$ & 0.5 & 0.2 & 0.3 & 0.1 & 0.6 \\
$f_{5}$ & 0.3 & 0.4 & 0.1 & 0.6 & 1
\end{tabular}

由上面的矩阵，按照聚类分析法得到相应的等价矩阵:

$$
R=\left(\begin{array}{lllll}
1 & 0.4 & 0.8 & 0.5 & 0.5 \\
0.4 & 1 & 0.4 & 0.4 & 0.4 \\
0.8 & 0.4 & 1 & 0.5 & 0.5 \\
0.5 & 0.4 & 0.5 & 1 & 0.6 \\
0.5 & 0.4 & 0.5 & 0.6 & 1
\end{array}\right)
$$

从实际情况出发, 若取 $\alpha=0.6$, 则可得到 $U$ 的分类为 $\left\{x_{1}, x_{3}\right\},\left\{x_{2}\right\}$ 和 $\left\{x_{4}, x_{5}\right\}$. 即 $x_{1}$ 与 $x$, 是 不易辨识的, $x_{4}$ 与 $x_{3}$ 也是不易辨识的. 这一结果与 Rough 集合理论中现有的结果相比显得 更为实用.

\section{$\Rightarrow$ 文 塥}

[1] Pawlak, Z., Stowinski, K., Stowinski, R., Int. J. Man-Machine Studies, 24(1986), 413-433.

[2]王国俊,中国科学, A 辑, 1983, 12: $1063-1072$.

[3] Pawlak, Z., Fuzzy Sets and Systems, 17(1985), 99-102.

[4] Shi, E. W., Fuzzy Sets and Systems, 23(1987), 387-391. 